\title{
Research Paper: The Relationship Between Mate Selection Styles and Marital Satisfaction of Married Nurses
}

\author{
Gholamreza Rajabi ${ }^{*}$, Ghodrat Allah Abbasi², Arash Jelodari ${ }^{1}$
}

1. Department of Counseling, Faculty of Education and Psychology, Shahid Chamran University of Ahvaz, Ahvaz, Iran.

2. Department of Psychology, Faculty Psychology, Sari Branch, Islamic Azad University, Sari, Iran.

Cltation: Rajabi, Gh, Abbasi, Gh. A., Jelodari A. (2016). The Relationship Between Mate Selection Styles and Marital Satisfaction of Married Nurses. Journal of Practice in Clinical Psychology, 4(4), 229-236. https://doi.org/10.18869/acadpub.jpcp.4.4.229

: https://doi.org/10.18869/acadpub.jpcp.4.4.229

\section{Article info:}

Received: 21 Apr. 2016

Accepted: 17 Aug. 2016

\section{Keywords:}

Mate-selection styles, Marital satisfaction, Nurses

\section{ABSTRACT}

Objective: This study was conducted to compare marital satisfaction among married nurses with three different mate selection styles.

Methods: This study is descriptive ex post facto. A total of 222 married nurses were selected using random sampling method from two educational hospitals affiliated to Jundishapur University of Medical Sciences and four private hospitals of Ahvaz, Iran. They completed mate selection styles questionnaire and the short form of marital satisfaction scale. The data were analyzed through ANOVA by using SPSS16.

Results: The findings indicated significant differences in marital satisfaction between married nurses with free choice and arranged styles and between married nurses with free choice and mixed styles. But there was no significant difference between married nurses with arranged and mixed styles with regard to their marital satisfaction.

Conclusion: The study nurses who belonged to recent generation of the community made their choices based on personal styles and criteria, and their families had no role in their marriage. Also, compared with last generations, they have higher marital satisfaction.

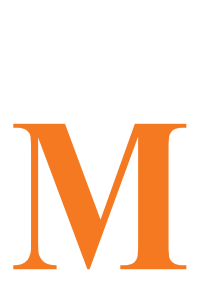

\section{Introduction}

arriage has been considered a sacred contract in many religions and different cultures; it has been regarded a moral and functional relationship between a man and woman, leading to the creation of a family and birth of children (Moshtagh, Teimourpour, \& Pourshanbaz, 2013; Sharma, Vaid, \& Kesar, 2005). Given the importance of marriage, the marital satisfaction or what helps people keep their mar- riage happy, have been widely studied (Larson \& Holman, 1994). The marital satisfaction is defined as the spouse's perception of his/her satisfaction of needs and desires by the other mate (Burpee \& Langer, 2005).

It helps preserving family unit and enjoying better quality of life, while dissatisfaction with marriage leads to anxiety, depression, and even dissolution of family (Anbari, Mohammadkhanai, \& Rezaei Dogaheh, 2014; Khaje Mansoori, Mohammadkhani, Mazidi, Kami, Bakhshi Nodooshan, \& Shahidi, 2016; Mohammadi, Farzinrad,

* Corresponding Author:

Gholamreza Rajabi, PhD

Address: Department of Counseling, Faculty of Education and Psychology, Shahid Chamran University of Ahvaz, Ahvaz, Iran.

Tel: +98 (61) 33333911

E-mail: rajabireza@scu.ac.ir 
Zargar, Ali, \& Behrooz, 2013; Delavar, Dolatshahi, Nouri, \& Ariyanfar, 2014; Skowron, 2000). Many studies have examined marital satisfaction during the last 15 years. These studies provide valuable information about marital satisfaction and factors affecting it such as commitment, spouse's support, individual characteristics, and so on (Bradbury, Fincham, \& Beach, 2000; Rostami, Veisi, Jafarian Dehkordi, \& Aalkasir, 2014; Shariatzadeh, Vaziri, \& Mirhashemi, 2014). However, an important issue which occupied researchers' minds is whether mate selection styles have impacts on marital satisfaction (Dinna, 2005).

Social scientists have studied different forms of marriage and mate selection. Researchers have generally introduced the free choice and the traditional or arranged mate selection styles (Dinna, 2005). The traditional mate selection is a marriage in which the parents or acquaintances interfere in the process of selecting the couple, but in free choice mate selection the couple meet each other and get married if they like each other. The traditional mate selection differs from the free choice mate selection (which ends in a contract between two persons) as it is a contract between two families. Before the initial session made by the family elders, the boy's parents investigate the girl's family background to make sure whether the girl's family belongs to the same or comparable economic category (Applbaum, 1995, cited in Dinna, 2005).

A review of the literature reveals that mate selection styles significantly differ in western and eastern cultures. In most western countries, people select freely their mates and usually in terms of their personal investigations and criteria like romantic love, sexual attractiveness, loneliness, intention to have children, and need to express the maturity. In contrast, in traditional communities, the choice is based on family connections, status, prestige, financial issues, basic skills, and health (Shachar, 1999). They believe that free choice marriages interfere the family bonds and impose coercion and pressure on family (Medora, Larson, Hortacsu, \& Dave, 2002).

Many studies indicate the change in marriage process, decrease in parental control over marriage, and increase in personal choice based on love (Ghimire, Axinn, Yabiku, \& Thornton, 2006). Heaton, Cammacj, and Young (2001) and also Hirschman and Teerawichitchainan (2003) reported that the traditional marriage has gradually decreased and the age of marriage has markedly increased in China and some Muslim countries of Southeast Asia. One reason for this change and increased free choice male selection in these societies, is the typical convergence model of modernity theory and the idea of modern human. In this regard, expansion of industrialization and urbanization, division of labor, formal education, employment in the factories, occupational and geographical mobility, and so on have provided structured conditions to live in a modern way which decreased reliance of the young people on families and choose a spouse more independently (Goode, 1963, cited in Zang, 2008).

With regard to different mate selection styles in the world, researchers have investigated the role of the styles in marital satisfaction and relationships. Very few studies have been conducted on mate selection styles based on factors other than romantic love (Myers, Madathil, \& Tingle, 2005). In general, there are contradictory results in this context. Blood research (1967) reported difference between the traditional (arranged) and free choice mate selection styles in term of marital satisfaction. He found that the marital satisfaction of people with the traditional marriage was higher than that of people with free choice and they experienced a happier life.

Myers et al. (2005) found no difference between marital and life satisfaction of people with free choice mate selection in America and people with traditional marriage in China. Yelsma and Athappilly (1988) found that the couples with traditional marriage had more marital satisfaction compared with the couples with free choice marriages in countries such as India and the United States. In contrast to these findings, Dinna (2005) found that the couples with free choice marriage had more marital satisfaction compared with the couples with traditional marriage. Xiaohe and Whyte (1990) also observed that the marital quality of the women with free choice mate selection was better than that in women with traditional marriage. Mazaheri, Sadeghi, Nasr Abadi, Ansari Nejad, and Abbasi (2009) reported that the "previous familiarity and marriage with the consent of the family" mateselection style and "woo and marry with the consent of the individual and the family" style had a higher level of marital adjustment compared with the styles of the "forced woo and marriage" and "previous familiarity with the spouse and forced marriage."

This research was conducted to investigate the role of mate selection styles as one of the factors causing marital dissatisfaction and divorce. Today, because of the ascending trend of divorce and marital dissatisfaction, social sciences and family psychology experts try to find the causes of divorce and its contributing factors. Although researchers have provided valuable information on successful marriage, marital satisfaction, and factors affecting it, there are few studies conducted on mate selection styles like traditional and free choice marriage 
and its contribution to marital satisfaction and divorce in our country.

Meanwhile, the social psychology literature has identified the idea of romantic love as the main factor of motivating individuals to form family in the recent years, and accordingly has introduced the notion of free mate selection, i.e. selecting mate without direct family intervention and solely based on both party's love, passion and desires. Thus another reason of conducting this research was to explore the role of love at the time of selecting partner in individuals' marital dissatisfaction.

Moreover, psychologists and marriage counselors can use the research results to help people enrich their knowledge and information on how to select a mate in premarital counseling and increase the effect of these factors in improvement of marital satisfaction after marriage. This study question was whether nurses with arranged, free choice, and mixed (a combination of arranged and free choice styles) mate selection styles felt different marital satisfaction.

\section{Methods}

This study is descriptive ex post facto. The statistical population comprised all male and female nurses of educational and private hospitals affiliated to Jundishapur Medical Sciences University of Ahvaz (1997 participants). To select the statistical sample, in the first stage, 4 private (Mehr, Ariya, Apadana, and Baghai) and 2 educational (Raazi and Shafa) hospitals were chosen randomly in the 3 districts of Ahvaz City. In the next stage, after coordination with nursing and security staff of hospitals, a full list of married nurses both official and contract employers was provided. Out of them and according to inclusion and exclusion criteria, 220 nurses were randomly selected with their full inform consent base on research ethics.

In the study sample, $58.1 \%$ (129) of nurses were males and $31.9 \%$ (93) were females; mean and standard deviation of female nurses' ages were 30.43 and 4.16 years, respectively. The duration of nurses' marriage duration ranged from 1 to 5 years; mean and standard deviation of their marriage duration were 3.17 and 0.37 years, respectively. Of these nurses, 74 had diploma, 41 junior college degree, 92 bachelor degree, 14 master degree, and $1 \mathrm{PhD}$ degree. A total of $89(40 \%)$ nurses married with arranged marriage, 57(26\%) with free choice marriage, and $76(34 \%)$ with mixed marriage.

The mate selection style questionnaire was designed to determine participants' mate selection style and ask them this question: "In general, how did you chose your partner?" The answer specified whether the participants chose their mates by their parents (arranged marriage), themselves and personal selection (free choice marriage), or themselves and the parents together (mixed marriage).

The marital satisfaction scale short-form was developed by Rajabi (2009) to measure marital satisfaction. It includes 13 items and rated on a 5-point Likert-type scale (1=disagree, to $5=$ totally agree). The items $3,4,6,8$, and 11 are scored inversely. The minimum and maximum scores are 13 and 65, respectively; high scores indicate high couples' marital satisfaction. Rajabi (2009)obtained the Cronbach $\alpha$ coefficient of the scale as 0.90 for the whole sample, 0.89 for men, and 0.91 for women, and its convergence validity coefficient with the 47-item ENRICH marital satisfaction questionnaire was significant $(\mathrm{P}<0.001)$.

In addition, Rajabi, Sarvestani, Khojaste Mehr, and Aslani (2013) reported the Cronbach $\alpha$ coefficient of the above scale as 0.89 and its validity with the 9-item life satisfaction questionnaire of Hills and Argyle (2001) as $0.29(\mathrm{P}<0.001)$. In the current study, the Cronbach $\alpha$ coefficient of the scale was 0.63 and its convergent validity coefficient with Sanai Zaker 42-item marital conflict questionnaire (1996) was $-0.35(\mathrm{P}<0.001)$.

In the current research, at first 6 hospitals in 3 districts of Ahvaz were randomly chosen and then with collaboration of chief of each hospital, a letter was sent to security department of each hospital indicating cooperation with researcher to distribute the questionnaires and select statistical samples. Then after coordination with security departments of hospitals and nursing staff, following a general interview and based on inclusion (being married, 1 to 5 years shared life experience, not having divorce or remarriage experiences) and exclusion (being single, less than 1 year shared life, having divorce or remarriage experiences) criteria, working nurses (official or contract) who have met the requirements of the research were included in the sample.

Furthermore, the interviews and questionnaire administration were done individually and with proper instructions in nurse stations and participants were reminded that their information and identities will be kept confidential to comply with ethical instructions. In this study, by using SPSS-16, we used the 1-way analysis of variance and the Scheffe post hoc test to study and compare mate selection styles among nurses.

\section{Results}

Because the sample size was more than 100, the data were normally distributed (Witte \& Witte, 2010) and the assump- 
tion of Levene's homogeneity of variance test of the dependent variable in three groups was met. The findings indicated that the variances of the three groups of free choice, arranged, and mixed marriage to the variable of marital satisfaction were equal $(\mathrm{P}=0.72, \mathrm{Z}=2.66)$. Regarding the normal distribution of data and the met assumption of equal variances, the ANOVA test could be used to compare three mate selection styles in terms of marital satisfaction.

As shown in Table 1, the F-ratio of ANOVA indicates that the people's marital satisfaction differs among three mate selection styles (arranged, free-choice, and mixed) $(\mathrm{P}<0.009, \mathrm{~F}=4.79)$. To determine which groups are different from each other, Scheffe post hoc test was used (Glass \& Stanley, 1970) (Table 2).

Based on Table 2, the marital satisfaction of nurses with arranged mate selection style is lower than nurses with free choice style $(\mathrm{P}<0.048,-2.12)$; also the marital satisfaction of nurses with free choice style is higher than nurses with mixed style $(\mathrm{P}<0.014,-2.62)$, and there is no significant difference between the marital satisfaction of nurses with arranged style and nurses with mixed style $(\mathrm{P}=0.819,0.49)$ (Figure 1).

\section{Discussion}

The findings of the current research indicate that how people select their partners can affect their marital satisfaction. In other words, the marital satisfaction of people who have selected their partners freely, i.e., based on personal choice, is higher than the other groups, namely arranged and mixed marriage ones. This finding confirms the results of other researchers (Xiaohe \& Whyte, 1990; Dinna, 2005; Gundogdu, 2007). Thus, young partners who select their mates independently and based on personal choice, tend to select mates through selection filters and premarital relationships. Selection filters may help them have more satisfaction than those who select their mates without sound familiarity (Shachar, 1999).

Dinna (2005) reported that the energy these people spend on mate selection, the years of familiarity, research, recognition and evaluation of future spouse, as

Table 1. The results of ANOVA of the mate selection styles in terms of marital satisfaction.

\begin{tabular}{ccccc}
\hline Mate Selection & $\mathbf{n}$ & Mean & SD & F \\
\hline Arranged & 89 & 32.69 & 5.49 & 4.79 \\
Free choice & 57 & 34.82 & 4.51 & 0.009 \\
Mixed & 76 & 32.19 & 4.91 & \\
\hline
\end{tabular}

PRACTICE in
CLINICAL PSYCH $Q$ LOGY

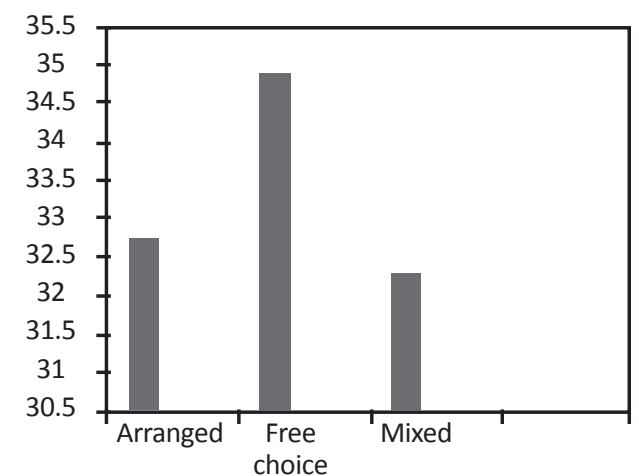

Marital satisfaction

Figure 1. The marital satisfaction in terms of mate selection styles.

Table 2. The results of the Scheffe test of marital satisfaction in terms of different mate selection styles.

\begin{tabular}{ccc}
\hline Groups Comparisons & Free Choice & Mixed \\
\hline Arranged & $-2.12(P<0.048)$ & $0.49(P=0.819)$ \\
Free choice & - & $-2.62(P<0.014)$ \\
\hline
\end{tabular}


compared to those who other people like parents choose their mates, results in increased marriage satisfaction in their life. Mosavi, Navabi Nejad, and Atef Vahid (2007) reported that marital conflicts in students who had premarital romantic relationships were lower than people who did not. Thus, couples' better understanding of each other after premarital relationships, familiarity with each other's personality, financial and family issues, would lead to their increased knowledge and thereby fewer conflicts in their shared future life.

In addition to selection filter, love in marriage is another good indicator for future marital satisfaction, whether in arranged marriage or free choice one. Various studies reported that most married people are healthier and happier (Bradbury et al., 2000). People who select their spouse based on free choice and personal decisions, adjust their most important priority for mate selection based on love and loyalty as leading and important factors in marriage and marital satisfaction. In marriages based on love, the couples are expected to obtain more information about each other's differences during their shared life (Myers et al., 2005).

There is no case reporting that low level of love has led to marital satisfaction. However, a marriage started with commitment can end with either success or failure. A marriage could not be successful when the couples have low emotional bonds with each other. In general, the level of love one feels in the beginning of marriage can be a good predictor for marital satisfaction in future. The level of love in the beginning of the marriage has great importance for the stability and satisfaction of couples (Dinna, 2005). According to our study results, love is an important and necessary element in marriage. Roizblatt, Kaslow, Rivera, Fuchs, Conejero, and Zacharias (1991) reported that love is one of the important components of marital satisfaction for most couples. However, most couples with arranged marriage do not start their family lives with love.

Therefore, the mate selection is an effective factor in marital satisfaction. This does not deny the role and importance of family in mate selection, rather appropriate and useful intervention of the family should be emphasized. An intervention in which the responsibility of decision making is upon the young people to freely select their spouse under the parents' supervision. Otherwise, by choosing their spouses independently and on the basis of romantic love, they may make irrational decisions in marriage. However, premarital trainings about mate selection styles and consequences of each one can be useful to resolve the problem.

Another finding indicated that the arranged marriage style was the most common style in the sample which included about $40 \%$ of participants. Aghajanian, Tashakori, and Mehryar (1996) reported that mate selection is a family and ethnic act rather than a personal act. These are the family and elders of the community that make decisions for the future of married couple. In this social context and ethnic diversity, inbreeding and consanguinity marriages are significantly more common than free choice mate selection, and except some cases, the opportunity of free choice mate selection is limited. Even if people initially meet each other for marriage, they would ask the parents and family about appropriateness of the person as a marital partner before any further attempts. This indicates that the family still has an important role in determining the appropriateness of a marital partner and their superiority over the children's mate selection issue is salient.

A review of the literature reveals that mate selection style can play an important role in establishing family's security and health, and it is clearly obvious that understanding these styles can be considered important for marriage and family counselors and psychologists in our community. Also it is a key factor to success in their premarital counseling to enrich and improve single people's information on mate selection and its effects on marital satisfaction after marriage.

The major limitation of the current study was the study sample who were nurses of Ahvaz City. So the results should be generalized to other populations with caution. It is recommended to conduct a longitudinal research to confirm the above findings using a large statistical population and a wider sample size among different cultures and cities in Iran. In addition to understanding the mate selection styles of Iranian society, we definitely found that mate selection styles have important role in individuals' marital satisfaction. Also, because of the role of mate selection in marital satisfaction, it is suggested that individuals be given enough premarital trainings about mate selection styles to be prepared for marriage.

\section{Acknowledgments}

We are sincerely thankful to all officials of the studied hospitals and nurses who cooperated with us in the study. The study was self-funded.

\section{Conflict of Interest}

The authors declared no conflict of interests. 


\section{References}

Aghajanian. A., Tashakori, A., \& Mehryar, H. A. (1996). Attitudes toward marriage, fertility, and labor market participation among adolescents in Iran. Paper presented at the Annual Meeting of the Population Association of America, New Orleans, USA March 29- April 21996.

Anbari, F., Mohammadkhani, P., \& Rezaei Dogaheh, E. (2014). Thought Control Strategies in Generalized Anxiety Disorder and Major Depressive Disorder and Their Relationships with Trait Anxiety. Practice in Clinical Psychology, 2(4), 229-36.

Blood, R. (1967). Love match and arranged marriage. New York: Free Press.

Bradbury, T., Fincham, F., \& Beach, S. (2000). Research on the nature and determinants of marital satisfaction: A decade in review. Journal of Marriage and the Family, 62(4), 964-80. doi: 10.1111/j.1741-3737.2000.00964.x

Burpee, L. C., \& Langer, E. J. (2005). Mindfulness and marital satisfaction. Journal of Adult Development, 12(1), 43-51. doi: 10.1007/s10804-005-1281-6

Delavar, B., Dolatshahi, B., Nouri, M., \& Aryanfar, K. (2014). Metacognition and depression, State Anxiety and Trait Anxiety Symptoms. Practice in Clinical Psychology, 2(3), 195-200.

Dinna, M (2005). Marital satisfaction in autonomous and arrange marriages: South African Indian sample (MA thesis). Pretoria: University of Pretoria.

Ghimire, D. J., Axinn W. G., Yabiku, S. T., \& Thornton, A. (2006). Social change, premarital nonfamily experience, and spouse choice in an arranged marriage society. American Journal of Sociology, 111(4), 1181-218. doi: 10.1086/498468

Glass, G. V., \& Stanley, J. C. (1970). Statistical methods in education and psychology. Englewood Cliffs, N. J.: Prentice-Hall Inc.

Gündoğdu, A. (2007). Relationship between self-construal's and marital quality (MA thesis). Ankara: Middle East Technical University.

Heaton, T. B., Cammacj, M., \& Young, L. (2001). Why is the divorce rate declining in Indonesia? Journal of Marriage and Family, 63(2), 480-90. doi: 10.1111/j.1741-3737.2001.00480.x

Hills, P., \& Argyle M. (2001). Emotional stability as a major dimension of happiness. Personality $\mathcal{E}$ Individual Differences, 31(8), 57-64. doi: 10.1016/s0191-8869(00)00229-4

Hirschman, C., \& Teerawichitchainan, B. (2003). Cultural and socioeconomic influences on divorce during modernization: Southeast Asia, 1940s to 1960s. Population and Development Review, 29(2), 215-53. doi: 10.1111/j.1728-4457.2003.00215.x

Khaje Mansoori, A., Mohammadkhani, P., Mazidi, M., Kami, M., Bakhshi Nodooshan, M., \& Shahidi, S. (2016). The Role of Metacognition and Intolerance of Uncertainty in Differentiating Illness anxiety and Generalized anxiety. Practice in Clinical Psychology, 4(1), 57-65.

Larson, J. H., \& Holman, T. B. (1994). Predictors of marital quality and stability. Family Relations, 43(2), 228-237. doi $10.2307 / 585327$

Mazaheri, A., Sadeghi, M. S., Nasr Abadi, O. L., Ansari Nejad, F., \& Abbasi, M. (2009). [Relationship of mate-selection styles with quality and stability of marriage (Persian)]. Journal of Culture Strategy, 5, 55-72.

Medora, N. P, Larson, J. H., Hortacsu, N., \& Dave, P. (2002). Perceived attitudes towards romanticism: A cross cultural study of American, Asian Indian, and Turkish young adults. Journal of Comparative Family Studies, 33(2), 155-182.

Mohammadi, A., Farzinrad, B., Zargar, F., Ali, M., \& Behrooz, B. (2013). Are metacognitive factors common in generalized anxiety disorder and dysthymia? Practice in Clinical Psychology, 1(3), 135-40.

Mosavi, S. S., Navabi Nejad, S., \& Atef Vahid, M. K. (2007) [The effects of premarital relationships on married students' marital conflict in universities of Tehran (Persian)]. Journal of Counseling Research (Innovations and Researches of Counseling), 6(22), 27-40.

Moshtaagh, N., Teimourpour, N., \& Pourshaanbaz, A. (2013) the relationship between attachment styles, marital satisfaction and Sex guilt with sexual desire in Iranian women. Practice in Clinical Psychology, 1(1), 17-24.

Myers, J. E., Madathil, J., \& Tingle, L. R. (2005). Marriage satisfaction and wellness in India and the United States: A preliminary comparison of arranged marriages and marriages of choice. Journal of Counseling $\mathcal{E}$ Development, 83(2), 183-90. doi: 10.1002/j.1556-6678.2005.tb00595.x

Rajabi, G, Sarvestani, Y., Khojaste Mehr, R., \& Aslani, K. (2013). [Predictors of marital satisfaction in married female nurses (Persian)]. Iran Journal of Nursing, 26(82), 23-33.

Rajabi, Gh. R. (2009). [Factorial structure of marital satisfaction scale in married staff of Shahid Chamran University of Ahvaz (Persian)]. Iranian Journal of Psychiatry and Clinical Psychology, 15(4), 351-58.

Roizblatt, A., Kaslow, F., Rivera, S., Fuchs, T., Conejero, C., \& Zacharias, A. (1999). Long lasting marriages in Chile. Contemporary Family Therapy: An International Journal, 21(1), 13-129. doi: 10.1023/a:1021918822405

Rostami, M., Veisi, N., Jafarian Dehkordi, F., \& Alkasir, E. (2014). Social Anxiety in Students with Learning Disability: Benefits of Acceptance and commitment therapy. Practice in Clinical Psychology, 2(4), 277-84.

Sanai Zaker, B. (1996). [Scales of assessment of marriage and family (Persian)]. Tehran: Be' esat Publication.

Shachar, R. (1999). His and her marital satisfaction: The double standard. Sex Roles, 25(7, 8), 451-67. doi: 10.1007/bf00292534

Shariatzadeh, M., Vaziri, S., \& Mirhashemi, M. (2014). comparison of early maladaptive schema in patients with dysthymic mood disorder, major depression and healthy control subjects. Practice in Clinical Psychology, 2(2), 91-100.

Sharma, N., Vaid, S. V., \& Kesar, A. (2005). Intergenerational differences in the concept of marriage among Dogra Brahmin females (Mothers and daughters). Anthropologist, 7(4), 253-56.

Skowron, E. (2000). The role of differentiation of self in marital adjustment. Journal of Counselling Psychology, 47(2), 229-37. doi: 10.1037/ / 0022-0167.47.2.229

Witte, R. S., \& Witte, J. S. (2010). Statistics (9 $9^{\text {th }}$ ed.). New York: John Willey Inc. 
Xiaohe, X., \& Whyte, K. J. (1990). Love matches and arranged marriages: A Chinese replication. Journal of Marriage and the Family, 52(3), 709-22. doi: 10.2307/352936

Yelsma, P., \& Athappilly, K. (1988). Marriage satisfaction and communication practices: Comparisons among Indian and American couples. Journal of Comparative Family Studies, 19, 37-54.

Zang, X. (2008). Gender and ethnic variation in arranged marriages in a Chinese city. Journal of Family Issues, 29(5), 615-38. doi: $10.1177 / 0192513 \times 07308039$ 
